\title{
Therapeutic effects of bone marrow-derived mesenchymal stem cells on radiation-induced lung injury
}

\author{
CHENGCHENG XIA ${ }^{1 *}$, PENGYU CHANG $^{1,2^{*}}$, YUYU ZHANG $^{1}$, WEIYAN SHI ${ }^{1}$, \\ BIN LIU ${ }^{3}$, LIJUAN DING ${ }^{1}$, MIN LIU ${ }^{1}$, LING GAO $^{1}$ and LIHUA DONG ${ }^{1}$
}

\begin{abstract}
${ }^{1}$ Department of Radiation Oncology, The First Bethune Hospital of Jilin University, Changchun, Jilin 130000; Key Laboratory of Radiobiology (Chinese Ministry of Health), School of Public Health, Jilin University, Changchun, Jilin 130000; ${ }^{2}$ Electrochemical State Key Laboratory, Changchun Institute of Applied Chemistry, Academy of Science, Changchun, Jilin 130000; ${ }^{3}$ Department of Orthopaedics, The First Bethune Hospital of Jilin University, Changchun, Jilin 130000, P.R. China
\end{abstract}

Received July 20, 2015; Accepted September 17, 2015

DOI: 10.3892/or.2015.4433

\begin{abstract}
Radiation-induced lung injury (RILI) is a fatal condition featured by interstitial pneumonitis and fibrosis. Mesenchymal stem cells (MSCs) have been widely used for treating RILI in rodent models. In the present study, we aimed to investigate whether the therapeutic effects of human bone marrow-derived mesenchymal stem cells (hBM-MSCs) on RILI were in a dose-dependent manner. A total of 100 mice were randomly divided into: a control group $(n=25)$, subject to lung irradiation and injection of phosphate-buffered solution (PBS) via the tail vein; and the hBM-MSC group, subject to lung irradiation followed by injection of a low dose $\left(1 \times 10^{3} \mathrm{hBM}-\mathrm{MSCs} / \mathrm{g}\right)$, medium dose $\left(5 \times 10^{3} \mathrm{hBM}-\mathrm{MSCs} / \mathrm{g}\right)$ and high dose $\left(1 \times 10^{4} \mathrm{hBM}-\mathrm{MSCs} / \mathrm{g}\right)$ of hBM-MSCs in PBS through the tail vein, respectively. After sacrifice, the pulmonary tissues were subject to hematoxylin and eosin (H\&E) staining, Masson's trichrome staining and immunohistochemical staining to investigate the pathological
\end{abstract}

Correspondence to: Professor Lihua Dong, Key Laboratory of Radiobiology (Ministry of Health), School of Public Health, Jilin University, 71 Xinmin Street, Chaoyang, Changchun, Jilin 130021, P.R. China

E-mail: drldong@163.com

*Contributed equally

Abbreviations: hBM-MSCs, human bone marrow-derived mesenchymal stem cells; HGF, hepatocyte growth factor; IL-1 $\alpha$, interleukin-1 $\alpha$; IL-1 $\beta$, interleukin-1 $\beta$; IFN- $\gamma$, interferon- $\gamma$; IL-10, interleukin-10; PBS, phosphate-buffered solution; PVE, portal vein embolization; PECAM, platelet endothelial cell adhesion molecule; RILI, radiation-induced lung injury; SPC, surfactant protein C; TNF- $\alpha$, tumor necrosis factor $\alpha$; TGF- $\beta 1$, transforming growth factor $\beta 1 ; \beta 2-\mathrm{MG}, \beta 2$ microglobulin

Key words: radiation pneumonitis, radiation pulmonary fibrosis, thoracic tumor, radiotherapy, bone marrow-derived mesenchymal stem cells changes. Immunofluorescent staining was performed to evaluate the differentiation capacity of hBM-MSCs in vivo by analyzing the expression of SPC and PECAM. hBM-MSCs improved the survival rate and histopathological features in the irradiated mice, especially in the low-dose group. Marked decrease in collagen deposition was noted in the irradiated mice treated using a low dose of hBM-MSCs. In addition, hBM-MSCs attenuated secretion and expression of IL-10 and increased the expression of TNF- $\alpha$. Furthermore, hBM-MSCs had the potential to differentiate into functional cells upon lung injury. Low-dose hBM-MSCs contributed to functional recovery in mice with RILI.

\section{Introduction}

Radiation-induced lung injury (RILI), in the form of pneumonitis and fibrosis, is fairly common in patients receiving thoracic radiation therapy. Unfortunately, the prognosis of patients with RILI is poor with a median survival time of less than 3 years $(1,2)$. Currently, extensive studies have been conducted to investigate the relationship between cytokines and the pathogenesis of RILI $(3,4)$. For example, overproduction of several cytokines appears to be related to the development of acute and late pulmonary toxicities after RILI, including pro-inflammatory cytokines such as interleukin-1 $\alpha$ (IL-1 $\alpha$ ), IL-1 $\beta$, interferon (IFN)- $\gamma$, IL-6, tumor necrosis factor $\alpha$ (TNF$\alpha$ ), and pro-fibrogenic cytokines such as transforming growth factor $\beta 1$ (TGF- $\beta 1$ ) and TGF- $\alpha$ (5-9).

Recently, accumulative evidence suggests that mesenchymal stem cells (MSCs), as gene therapy delivery vehicles, are involved in the repair of lung tissue damage by differentiating into functional cells and facilitating lung tissue regeneration by means of the generation of cytokines $(10,11)$. For example, hepatocyte growth factor gene-modified MSCs contributed to the attenuation of RILI and inhibition of lung fibrosis (12). In addition, Asmussen et al (13) reported that human MSCs contributed to the improvement of oxygenation and attenuation of pulmonary oedema, especially in a high-dose group. Moreover, Devaney et al (14) revealed that a high dose of hMSCs was most effective in reducing $E$. coli-induced lung 
injury compared with a low-dose group. In the present study, we hypothesized that the therapeutic potential of bone marrow (BM)-derived MSCs on RILI may function in a dose-dependent manner. To this end, three different doses of hBM-MSCs were administrated in a mouse model of RILI to evaluate the therapeutic effects of different doses of hBM-MSCs in vivo. Our results revealed that low-dose hBM-MSCs contributed to functional recovery in mice with RILI.

\section{Materials and methods}

Cell culture. hBM-MSCs, kindly provided by the Cancer Center of the First Bethune Hospital of Jilin University (Changchun, China), were seeded into a flask with basal MSC medium supplemented with 5\% fetal bovine serum (FBS), and $1 \%$ mesenchymal stem cell growth supplement (MSCGS) and $1 \%$ penicillin/streptomycin (Biowit Technologies, Shenzhen, China). Subsequently, the cells were cultured at $37^{\circ} \mathrm{C}$ with $5 \%$ $\mathrm{CO}_{2}$ in a humidified atmosphere. Passaging was conducted every 2-3 days, and cells of passage 5 (P5) were used in the present study.

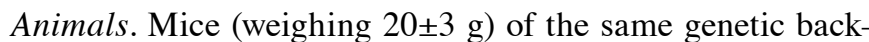
ground were purchased from HFK Bioscience Co., Ltd. (Beijing, China). All animals were maintained under specific pathogen-free conditions, and had free access to water and a standard rodent diet provided by the Animal Center of Jilin University (Changchun, China). The study protocols were approved by the Animal Care and Use Committee of the Chinese Academy of Medical Sciences (Beijing, China).

Induction of RILI. To establish the RILI model, the whole lung was exposed to irradiation with a dose rate of $1,500 \mathrm{mGy} / \mathrm{min}$ using a low pass energy X-RAD 320 X-ray system (Precision X-ray; North Branford, CT, USA). The total dose was $18 \mathrm{~Gy}$. The voltage was set at $300 \mathrm{kV}$, and the current was $11.79 \mathrm{~mA}$.

Experimental design. A total of 100 mice were randomly divided into: i) a control group $(n=25)$, which was subject to lung irradiation followed by injection of phosphate-buffered solution (PBS) via the tail vein; ii) a low-dose hBM-MSC group which was subject to lung irradiation followed by injection of $1 \times 10^{3} \mathrm{hBM}-\mathrm{MSC} / \mathrm{g}$ in PBS through the tail vein; iii) a medium-dose hBM-MSC group which was subject to lung irradiation followed by $5 \times 10^{3} \mathrm{hBM}-\mathrm{MSCs} / \mathrm{g}$ through the tail vein; and iv) a high-dose hBM-MSC group which was subject to lung irradiation followed by $1 \times 10^{4} \mathrm{hBM}-\mathrm{MSC} / \mathrm{g}$ through the tail vein. The delivery of hBM-MSCs was performed within $24 \mathrm{~h}$ after radiation.

Three mice randomly selected in each group were sacrificed under euthanasia on day 3, 7, 14, 28 and 84 after irradiation. Eye venous blood was collected to determine the cytokine levels using enzyme-linked immunosorbent assay (ELISA).

ELISA. Cytokine levels including HGF, IL-10 and TGF- $\beta 1$, and $\mathrm{Col}$ were measured using standard ELISA kits purchased from R\&D Systems (Minneapolis, MN, USA), eBioscience (San Diego, CA, USA) and USCN (Wuhan, China), according to the manufacturer's instructions.
Flow cytometry. Flow cytometry was carried out to determine the expression of cell-surface markers of the MSCs. Cells (P5) were fixed for $30 \mathrm{~min}$ in ice-cold $1 \%$ paraformaldehyde. Afterwards, the cells were stained at room temperature in the dark with the following primary mouse anti-human antibodies purchased from BD Biosciences (Franklin Lakes, NJ, USA): PE-conjugated CD11b, FITC-conjugated CD19, FITC-conjugated CD34, PE-conjugated CD45, PE-conjugated CD73, PE-conjugated CD90, PE-conjugated CD105 and PE-conjugated HLA-DR. FITC- or PE-conjugated mouse IgG1 served as the isotype control.

Tri-lineage differentiation. Cells (P5) were induced to differentiate into adipocytes, osteoblasts and chondrocytes using the StemPro ${ }^{\circledR}$ Adipogenesis differentiation kit, StemPro ${ }^{\circledR}$ Osteogenesis differentiation kit, and StemPro ${ }^{\circledR}$ Chondrogenesis differentiation kit (Life Technologies, Carlsbad, CA, USA) according to the manufacturer's instructions. The medium was replaced every 2-3 days. Approximately 21 days later, cell identification was performed by staining with Red Oil O, Alizarin Red and Aniline Blue, respectively.

Histological analysis. The left lungs were fixed using 10\% neutral formalin for 8-10 h, followed by embedding with paraffin. Then the sections $(4-\mu \mathrm{m})$ were subjected to hematoxylin and eosin (H\&E) staining, Masson's trichrome staining and immunohistochemical staining, respectively. The images were evaluated by two qualified staff blinded to the details of this study using an Olympus BX51 microscope (Olympus, Tokyo, Japan).

Real-time PCR analysis. Total RNA was extracted from lung tissues using TRIzol (Invitrogen Inc., Carlsbad, CA, USA). The cDNA synthesis of TNF- $\alpha$ was performed using $1 \mu \mathrm{g}$ RNA using M-MLV reverse transcriptase (Takara, Shiga, Japan). Real-time PCR amplification was carried out using SYBR on a Life 7500 Fast system (Life Technologies) with the following primers: TNF- $\alpha$, 5'-ATCCgCgACgTggAACTg-3' and 5'-ACCgCCTggAgTTCTggAA-3'; and $\beta$-actin, 5'-TGAGC TGCGTTTTACACCCT-3' and 5'-AGGGTGAGGGACTTCC TGTAA-3'. PCR reactions were performed using a total of $20 \mu \mathrm{l}$ containing $10 \mu \mathrm{l} 2 \mathrm{X}$ SYBR Premix, $0.6 \mu \mathrm{l}$ of each specific primer to a final concentration of $300 \mathrm{nM}$, and $1 \mu \mathrm{l} \mathrm{cDNA}$ template. PCR was performed under the following conditions: degeneration at $95^{\circ} \mathrm{C}$ for $15 \mathrm{~min}$, followed by 40 cycles of denaturation at $95^{\circ} \mathrm{C}$ for $10 \mathrm{sec}$, annealing at $65^{\circ} \mathrm{C}$ for $30 \mathrm{sec}$ and extension at $72^{\circ} \mathrm{C}$ for $30 \mathrm{sec}$. The mRNA level was normalized by $\beta$-actin. The amplification results were calculated as $2^{-\Delta \Delta \mathrm{Ct}}$ according to a previous description (15).

Immunofluorescent staining. Immunofluorescent staining was performed to evaluate the tri-lineage differentiation capacity of hBM-MSCs in vivo by analyzing the expression of SPC and PECAM. In this section, 10 immunodeficiency mice were randomly divided into a non-radiation + hBM-MSC group, subject to injection of $1 \times 10^{4} \mathrm{hBM}-\mathrm{MSC} / \mathrm{g}$ in PBS through the tail vein; and a lung irradiation $+\mathrm{hBM}-\mathrm{MSC}$ group, subject to lung irradiation followed by injection of $1 \times 10^{4} \mathrm{hBM}-\mathrm{MSC} / \mathrm{g}$ in PBS through the tail vein. The animals were sacrificed 56 days after injection, and the left lung was collected. Subsequently, 
A

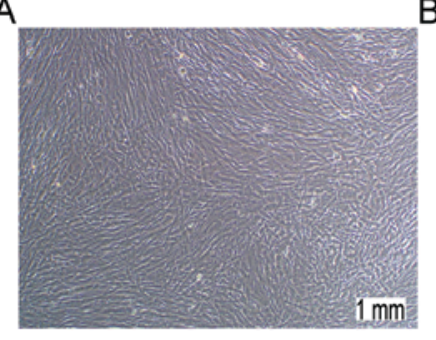

B

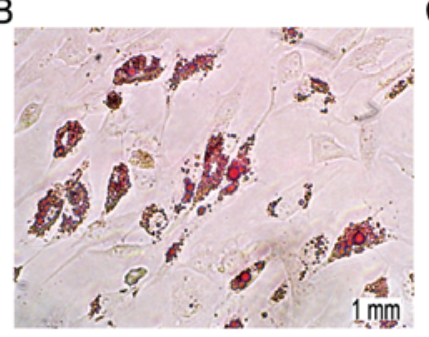

C

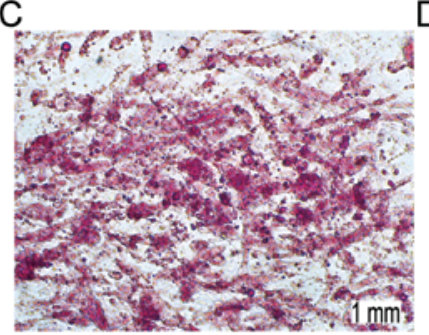

D

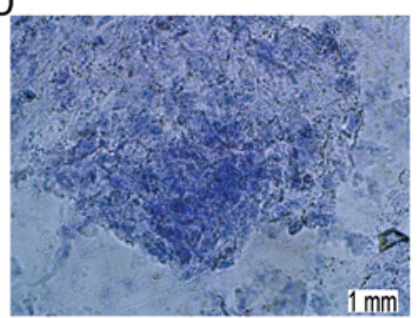

E
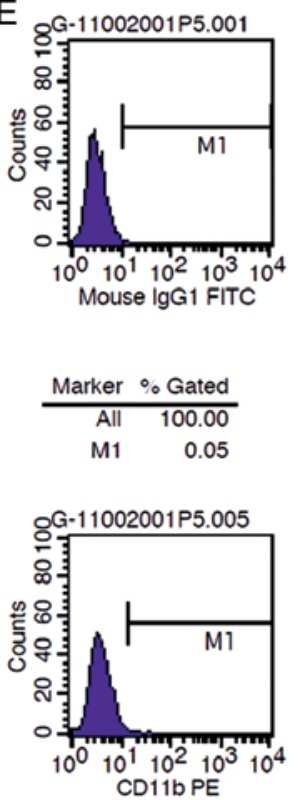

\begin{tabular}{rr} 
Marker & $\%$ Gated \\
\hline All & 100.00 \\
M1 & 0.38
\end{tabular}

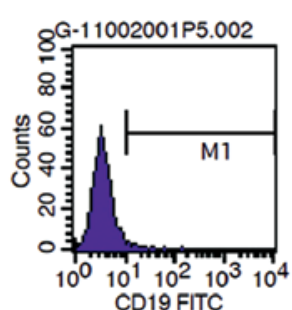

Marker $\%$ Gated

$\begin{array}{rr}\text { All } & 100.00 \\ \text { M1 } & 0.99\end{array}$
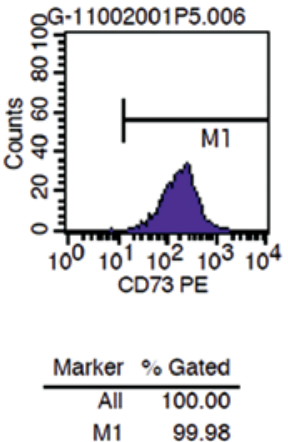

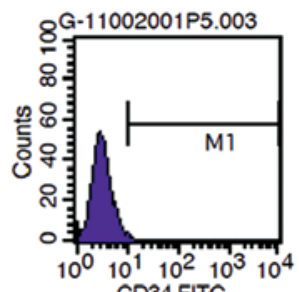

CD34 FITC
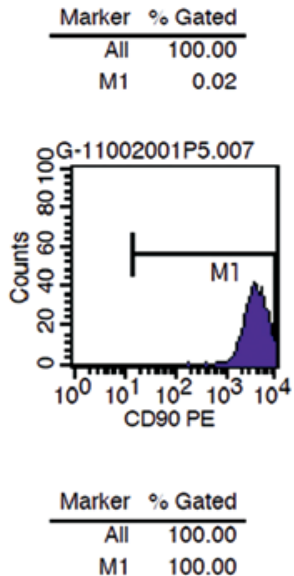
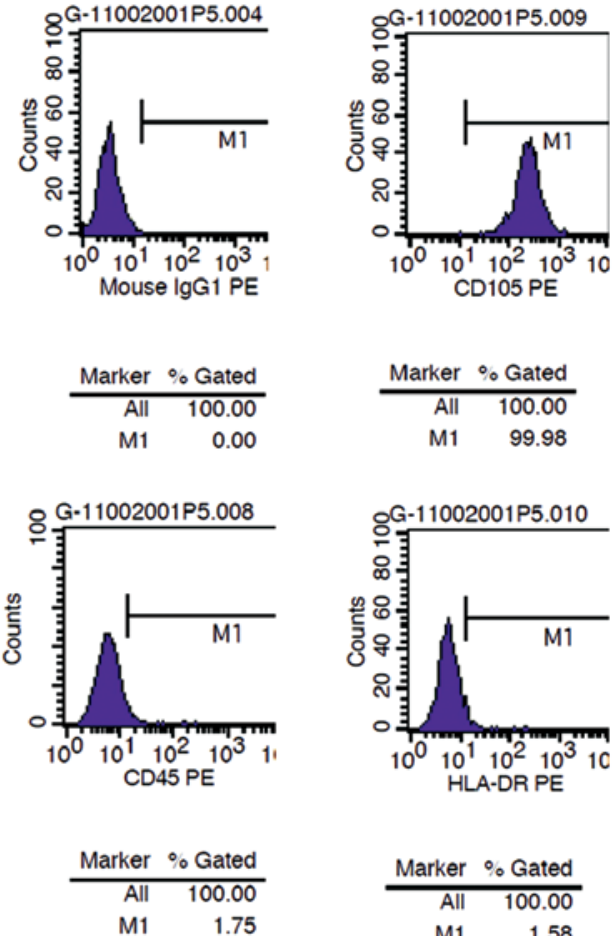

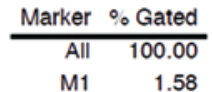

Figure 1. Morphology and features of hBM-MSCs. (A) The hBM-MSCs exhibited a spindle-like shape under in vitro culture. (B-D) Tri-lineage differentiation of hBM-MSCs. hBM-MSCs had the capacity to differentiate into adipocytes (B), osteoblast (C) and chondrocytes (D). (E) Quantitative analysis of hBM-MSCs by FACS. Scale bar, $1 \mu \mathrm{m}$.

the tissues were dewaxed in xylene and rehydrated with a gradient series of ethanol. Thereafter, the sections were incubated with the primary antibody against $\beta 2$ microglobulin, SPC and PECAM at $4^{\circ} \mathrm{C}$ overnight, followed by incubation with the secondary antibodies for $2 \mathrm{~h}$ at room temperature. The images were observed using PerkinElmer UltraView Vox confocal microscopy (PerkinElmer, Waltham, MA, USA).

Statistical analysis. Data are presented as the mean \pm standard deviation. Statistical evaluation was performed using analysis of variance with a Tukey's post hoc test. $\mathrm{P}<0.05$ was indicative of a significant difference.

\section{Results}

Morphology and features of hBM-MSCs. Under in vitro conditions, hBM-MSCs are characterized by their spindle-like shape and clear cellular boundaries (Fig. 1A). In addition, hBM-MSCs show the capability of differentiating into adipocytes, osteoblasts and chondrocytes after culturing in defined medium (Fig. 1B-D). Cell-surface marker analysis showed that the hBM-MSCs represented a population of cells with expression of CD73, CD90 and CD105, and absence of hemapoietic markers such as CD11b, CD19, CD34 and CD45 (Fig. 1E).

Low-dose hBM-MSCs improve the survival rate and histopathological features in irradiated mice. The survival rate of animals in the hBM-MSC treatment groups was higher than the rate in the irradiation alone group. The survival rate of the mice in the low-dose MSC group was higher than that of mice in the high-dose group (Fig. 2A). For the histopathological results, RILI-associated features were initially observed on day 3 and were characterized by degradation of capillaries within alveolar septa and extravasation of erythrocytes into alveolar spaces (Fig. 2B). On day 28, alveolar structures with pathological lesions were observed in the lung with infiltration of inflammatory cells in the interstitial part and abnormal stromal hyperplasia. On day 84 , infiltration of inflammatory cells and severe interstitial hyperplasia were observed. Compared with the control group, reduction in airspace inflammation and alveolar hemorrhage was observed in the groups treated with hBM-MSCs. In addition, the thickness of the interalveolar septa was modestly increased in the control 

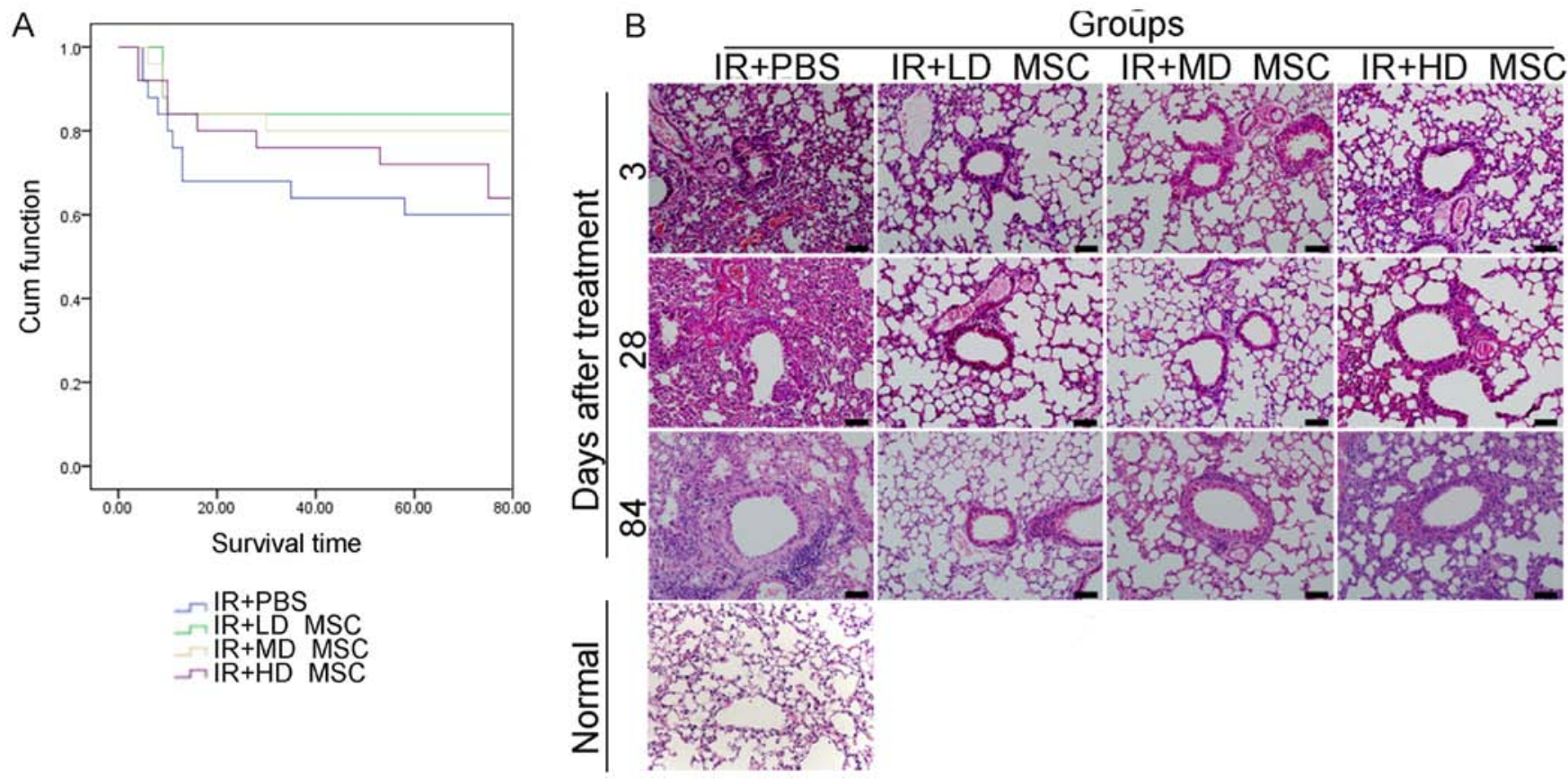

Figure 2. Effects of hBM-MSCs on radiation-induced lung injury. (A) hBM-MSCs prolonged the survival period of irradiated (IR) mice compared with the control group. (B) hBM-MSCs contributed to the maintenance of pulmonary alveoli compared with the irradiation group. In addition, infiltration of inflammatory factors and matrix apposition was markedly induced in the hBM-MSC group, especially in the low-dose group. IR + PBS, irradiation alone group; IR+LD MSC, irradiation + low-dose MSC group; IR+MD MSC, irradiation + middle-dose MSC group; IR+HD MSC, irradiation + high-dose MSC group. Scale bar, $20 \mu \mathrm{m}$.

group. Whereas, a slight reduction was noted in the thickness of the interalveolar septa in the high-dose group, while a significant reduction was noted in that of the low-dose group. These features may be responsible for the differences in the survival rate of the mice in each group.

hBM-MSCs decrease the collagen deposition in radiation lung injury. Masson staining on day 28 showed alveolar, bronchial, and vascular collagen deposition, which was increased on day 84 (Fig. 3A). Treatment of hBM-MSCs contributed to a decrease in collagen deposition in the radiation lung injury, especially in the low-dose group.

The expression of HGF and TGF- $\beta 1$ in the stroma was aberrant after radiotherapy (Fig. 3B). After radiation, HGF expression was downregulated in blood vessels and bronchus. TGF- $\beta 1$ was not expressed in normal lung tissues. Marked upregulation of TGF- $\beta 1$ expression was noted in the radiation group; however, its expression was significantly decreased in the hBM-MSC groups compared with the radiation alone group.

HGF and TGF- $\beta 1$ protein concentrations in peripheral blood were measured by ELISA (Fig. 3C and D). Serum TGF- $\beta 1$ was reduced on day 28 and 84 after hBM-MSC interference. Compared with the non-treatment group, the serum HGF was increased after hBM-MSC interference on day 3, 7, 14, 28 and 84, respectively $(\mathrm{P}<0.05)$. Regarding Colla3 protein, the expression of Col was downregulated in the lung tissue on day 84 after hBM-MSC interference compared with the control group (Fig. 3E). Among the hBM-MSC groups, the expression of Col was significantly reduced in the low-dose group compared with the middle- and high-dose groups, respectively.
hBM-MSC therapy attenuates the secretion and expression of pro-inflammatory cytokines and improves the expression of anti-inflammatory cytokines. To evaluate the anti-inflammatory activity of hBM-MSCs, IL-10 was measured by ELISA in peripheral blood on day 3, 28 and 84 after radiation (Fig. 4A). The results indicated that hBM-MSCs significantly increased the expression of IL-10.

Real-time RT-PCR was used to detect the local inflammatory reaction of lung tissue on day 28 after radiation. Compared with the radiation group, hBM-MSCs reduced the expression of TNF- $\alpha$ mRNA (Fig. 4B).

hBM-MSCs differentiate into functional cells in the presence of lung injury. In order to prove the differentiation potential of hBM-MSCs, 10 immunodeficiency mice were divided into a non-radiation plus hBM-MSC (1x10 $\left.{ }^{4} \mathrm{hBM}-\mathrm{MSCs} / \mathrm{g}\right)$ group, and a lung irradiation plus hBM-MSC $\left(1 \times 10^{4} \mathrm{hBM}-\mathrm{MSCs} / \mathrm{g}\right)$ group, respectively. The epithelial cell markers, SP-C, and hBM-MSC marker, $\beta 2$ microglobulin $(\beta 2-\mathrm{MG})$, were co-expressed in the lung irradiation plus hBM-MSC group (Fig. 5A). In addition, endothelial cell markers, PECAM and $\beta 2-\mathrm{MG}$, were co-expressed in the lung irradiation plus hBM-MSC group (Fig. 5B). In contrast, such markers were not expressed in the non-radiation plus hBM-MSC group. Taken together, the differentiation capacity of hBM-MSCs into functional cells only occured in the presence of lung damage.

\section{Discussion}

In the present study, we hypothesized that the therapeutic potential of BM-MSCs on RILI may occur in a dose-dependent manner. Our results revealed that BM-MSCs could attenuate 
A

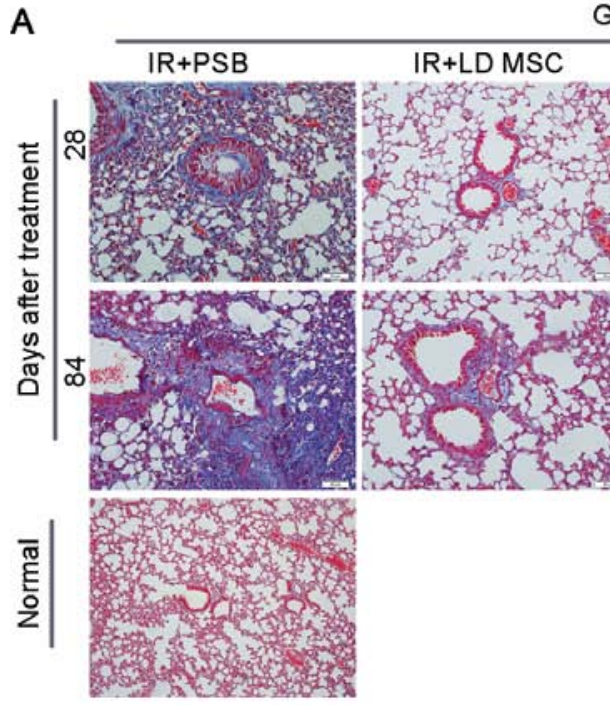

B

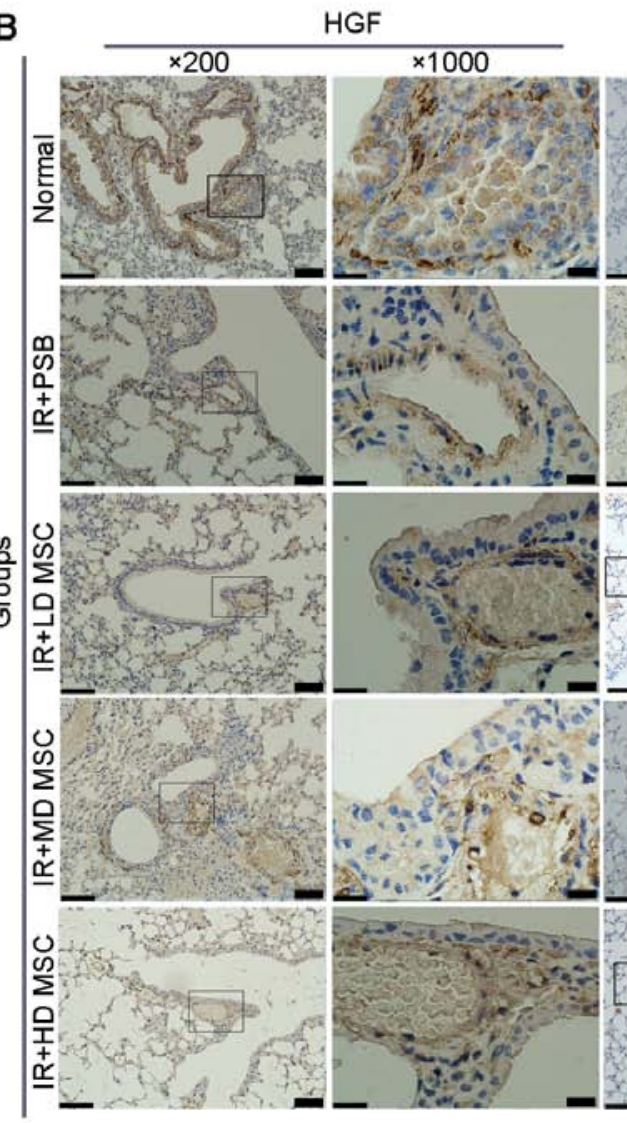

Groups

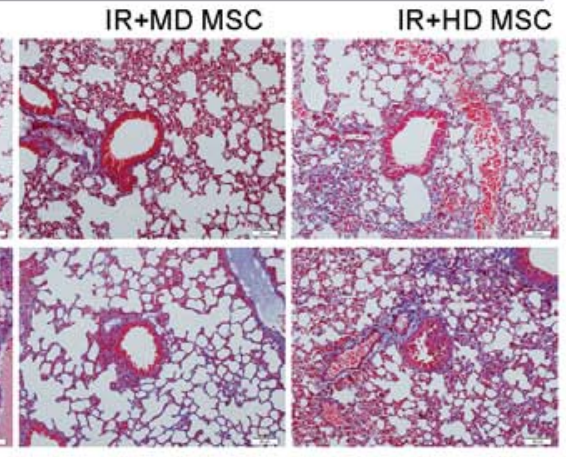

IR+MD MSC

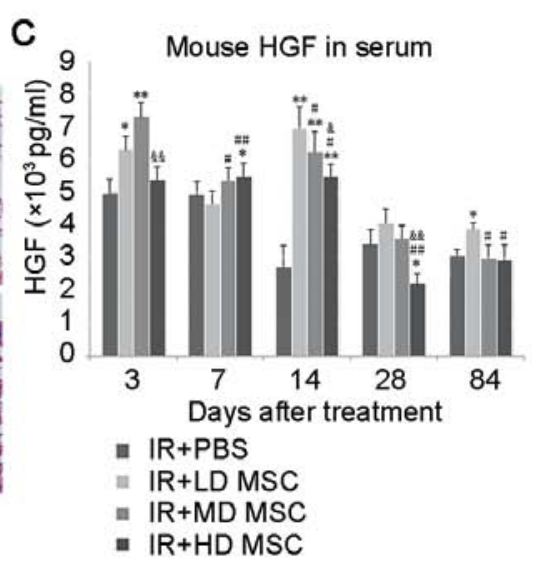

- IR+PBS

= IR+LD MSC

- IR+MD MSC

- $\mathrm{IR}+\mathrm{HD} M S C$

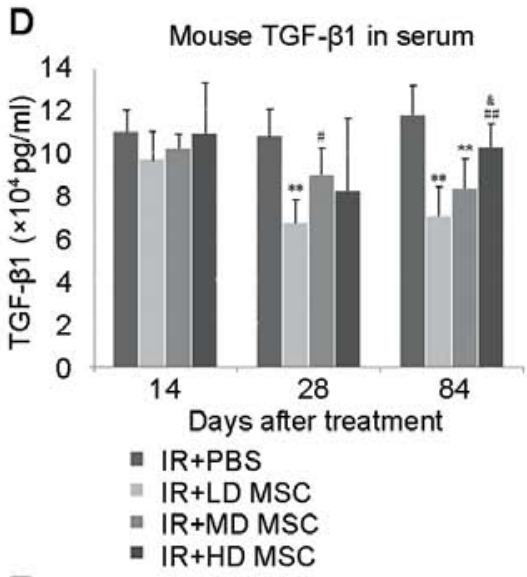

E

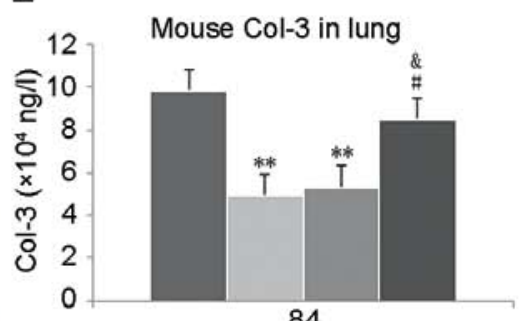

Days after treatment

Figure 3. Expression of fibrosis-related markers in each group. (A) Collagen deposition was markedly induced in mice following administration of hBM-MSCs, especially in the low-dose group, compared with the irradiation alone group. (B) Overexpression of HGF was observed in normal mice, while its expression was significantly decreased in the irradiation alone group. However, after interference of hBM-MSCs, the expression of HGF was increased compared with the irradiation alone group. hBM-MSCs inhibited the expression of TGF- $\beta 1$ compared with the irradiation group, indicating its role in the inhibition of fibrosis in vivo. (C-E) Expression levels of hepatocyte growth factor (HGF), transforming growth factor (TGF)- $\beta 1$ in serum and Col-3 in lung tissues after treatment were measured by ELISA. ${ }^{*} \mathrm{P}<0.05$ vs. the radiation group; ${ }^{* *} \mathrm{P}<0.01$ vs. the radiation group; ${ }^{*} \mathrm{P}<0.05$ vs. the low-dose MSC group. ${ }^{\# /} \mathrm{P}<0.01$ vs. the low-dose MSC group. ${ }^{\&} \mathrm{P}<0.05$ vs. middle-dose MSC group; ${ }^{\&} \mathrm{P}<0.01$ vs. middle-dose MSC group. IR+PBS, irradiation alone group; IR+LD MSC, irradiation + low-dose MSC group; IR+MD MSC, irradiation + middle-dose MSC group; IR+HD MSC, irradiation + high-dose MSC group. Scale bar, $20 \mu \mathrm{m}$.

RILI in mice compared with a control group. The survival rate of the mice in the low-dose MSC group was higher than that of mice in the high-dose group.

Previous studies have investigated the protective effects of clinical grade hMSCs under in vivo conditions using a low dose and a high dose, respectively. These results revealed that different doses of MSCs could exert protective effects in vivo compared with the control groups. Interestingly, the low-dose group was proven to be the more effective in improving the functional properties compared with the high-dose groups (13,16-19). In addition, a higher incidence of adverse events may occur in the high-dose group. For example, Li et al (20) showed that the 
A

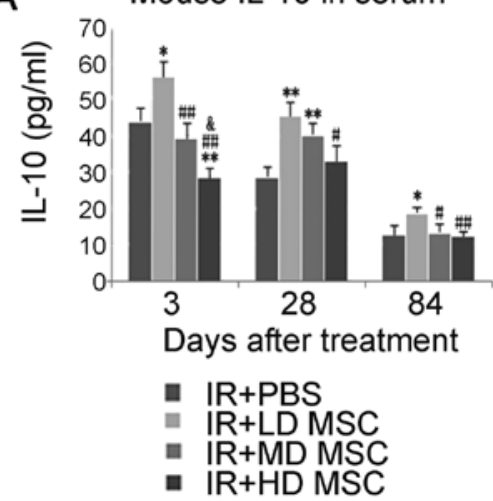

B TNF- $\alpha$ expression in lung

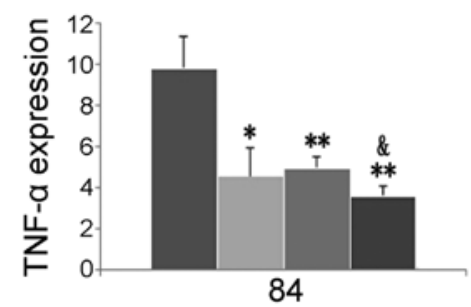

Days after treatment

- IR+PBS

- IR+LD MSC

- IR+MD MSC

- IR+HD MSC

Figure 4. Expression levels of pro-inflammatory and anti-inflammatory cytokines in peripheral blood and lung tissue. (A) A significant increase was noted in the levels of IL-10 in the hBM-MSC groups compared with the control group, especially in the low-dose group. " $\mathrm{P}<0.05$ vs. radiation group; ${ }^{* *} \mathrm{P}<0.01$ vs. radiation group. ${ }^{~} \mathrm{P}<0.05$ vs. low-dose MSC group; ${ }^{\# \#} \mathrm{P}<0.01$ vs. low-dose MSC group. ${ }^{\circledR} \mathrm{P}<0.05$ vs. middle-dose MSC group. (B) TNF- $\alpha$ mRNA expression was significantly decreased in the hBM-MSC groups compared with the control group. ${ }^{*} \mathrm{P}<0.05$ vs. radiation group; ${ }^{* *} \mathrm{P}<0.01$ vs. radiation group; ${ }^{*} \mathrm{P}<0.05$ vs. middle dose MSC group. ${ }^{\&} \mathrm{P}<0.01$ vs. low dose MSC group. IR+BS, irradiation alone group; IR+LD MSC, irradiation + low-dose MSC group; IR+MD MSC, middle-dose MSC group.

A
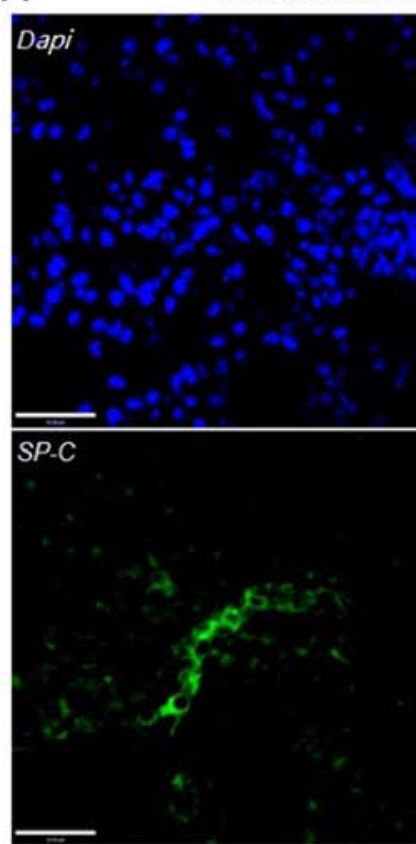

Differentiation into type II AEC

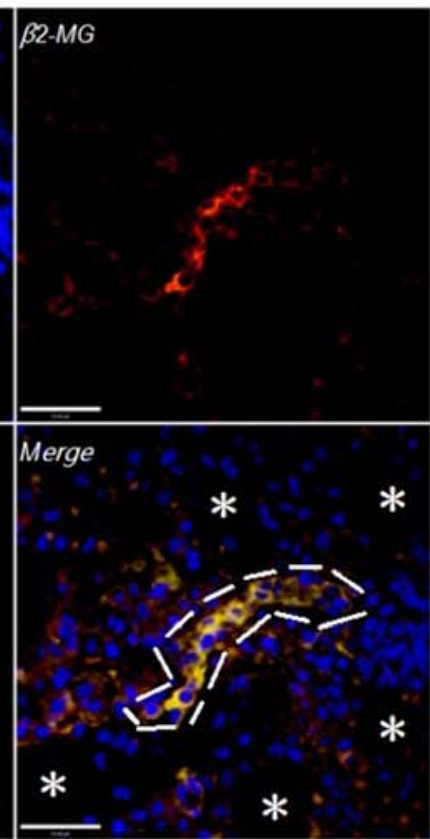

B
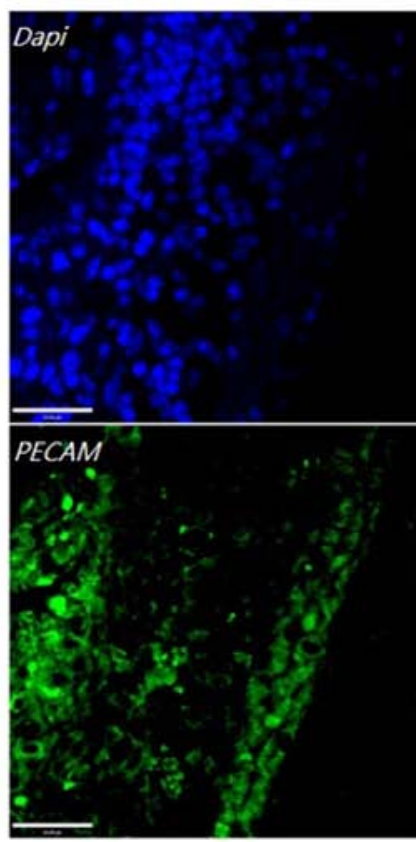

Differentiation into endothelial cells

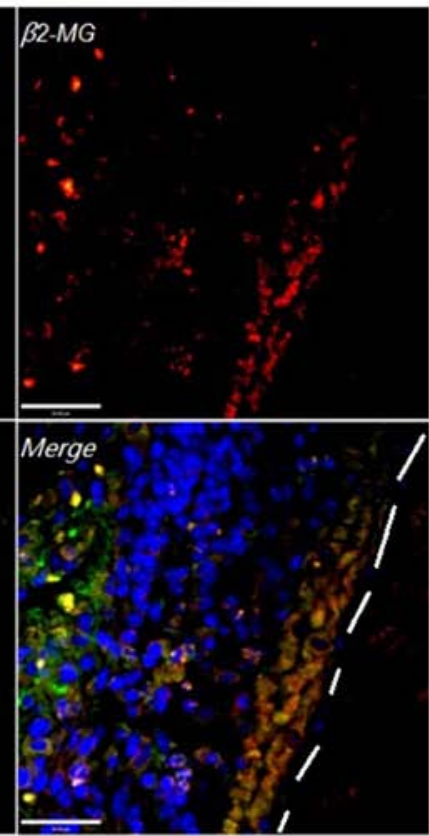

Figure 5. hBM-MSCs can differentiate into vascular endothelial cells and alveolar epithelial cells in the irradiated lung. (A) Double staining of SPC/ $\beta 2$-microglobulin ( $\beta 2-\mathrm{MG}$ ). Blue color, cell nucleus; green color, alveolar epithelial cell marker SPC; red color, $\beta 2-\mathrm{MG}$. (B) Double staining of PECAM/ $\beta 2-\mathrm{MG}$. Blue color, cell nucleus; green color, vascular endothelial cell marker PECAM; red color, $\beta 2-\mathrm{MG}$. The images were observed at a magnifications of x400. Scale bars, $33 \mu \mathrm{m}$.

high-dose $\left(1.0 \times 10^{6}\right.$ and $\left.5.0 \times 10^{5}\right)$ groups induced lethal portal vein embolization (PVE) in mice with liver disease. On the contrary, no PVE and related death was observed in the lowdose $\left(2.5 \times 10^{5}\right)$ group. Similarly, a low dose of MSCs showed greater safety and better therapeutic effects for RILI compared with the high-dose group. Compared with the control group, the hMSC treatment groups experienced alleviation of RILI, especially in the low-dose group.

To investigate the potential mechanism of the therapeutic effects of MSCs involved in RILI, we firstly assessed the expression of fibrosis-related factors TGF- $\beta 1$, HGF and Col.
TGF- $\beta 1$, a major mediator involved in pro-inflammatory responses and fibrotic tissue remodeling, has been considered to play a crucial role in RILI (21). Meanwhile, inflammation may promote the expression of TGF- $\beta 1$, forming a vicious circle in RILI. In the present study, the expression of TGF- $\beta 1$ in the treatment groups was markedly downregulated compared with the irradiation group on day 28 and 84 after hBM-MSC interference, indicating that HBM-MSCs play important roles in the inhibition of fibrosis. HGF, associated with mitogenic, morphogenic and anti-apoptotic activities of BM-MSCs, was found to enhance the regeneration of the lung and to have an 
inhibitory effect on fibrosis (22). Wang et al (12) reported that Ad-HGF-modified MSCs improved histopathological and biochemical markers of RILI by attenuating the expression of inflammatory factors and fibrosis factors (e.g., TGF- $\beta$ ) and inhibiting fibrotic progression. In addition, compared with the non-transfected MSC group, Ad-HGF-modified MSCs contributed to the improvement of RILI by enhancing the release of endogenous HGF. Moreover, in a previous study, Kim et al (23) reported that $\mathrm{MSC} / \mathrm{HGF}$ interference resulted in a significant reduction in liver fibrosis associated with elevated HGF levels and decreased TGF- $\beta 1$ after MSC/HGF therapy. In the present study, a significant difference was noted in the expression of HGF after hBM-MSC interference compared with the control group on day 3,14 and 28, especially the low-dose hBM-MSC group. Based on these results, we concluded that HGF plays an important role in the inhibition of fibrosis in RILI.

Next, we assessed the effects of BM-MSCs on inflammation-related factors such as TNF- $\alpha$ and IL-10. Previous studies revealed that murine MSCs could respond to pulmonary injury, reduce pro-inflammatory cytokines (IL-6 and TNF- $\alpha$ ), collagen deposition and increase the expression levels of IL-10 and PGE2 (10,24-28). In addition, Lee et al showed that MSCs could restore alveolar fluid clearance, reduce inflammation, and exert antimicrobial activity partly through secretion of keratinocyte growth factors (29). In our study, hBM-MSCs appeared to be mediated by a shift from a pro-inflammatory response to RILI, and contributed to the alleviation of radioactive lung injury. Compared with the control group, downregulation of TNF- $\alpha$ and upregulation of IL-10 were observed in the BM-MSC group, especially the low-dose hBM-MSC group.

BMSCs have been reported to have the capacity to differentiate into various cell types, including endothelial cells, epithelial cells (30), adipocytes and osteocytes (31-33). In the present study, infrequent BM-MSC engraftment resulted in differentiation of BM-MSCs into epithelial cells and endothelial cells as revealed by double immunofluorescence staining. Furthermore, few MSCs were observed within the injured sites compared to tremendous loss of functional cells in the impaired tissues. Thus, we speculate that the tissue regeneration may not largely depend on direct differentiation of MSCs into functional cells. Considering the fact that therapeutic effects were observed even though heterogenic MSCs were rapidly cleared by the host after transplantation, we conclude that BM-MSCs facilitated tissue repair mainly though paracrine or autocrine actions.

In conclusion, a low dose of hBM-MSCs is superior to a high dose of hBM-MSCs with excellent safety and differentiation capacity in mice with RILI. We speculate that hBM-MSCs attenuate lung injury mainly via paracrine mechanisms, including upregulation of HGF and IL-10, as well as downregulation of TNF- $\alpha$, TGF- $\beta 1$ and Col- 3 . Thus, low-dose hMSCs may be a promising therapeutic approach for the management of RILI.

\section{Acknowledgements}

This study was supported by grants from the National Natural Science Foundation of China (grant nos. 81272999 and 81372929).

\section{References}

1. Mehta V: Radiation pneumonitis and pulmonary fibrosis in non-small-cell lung cancer: Pulmonary function, prediction, and prevention. Int J Radiat Oncol Biol Phys 63: 5-24, 2005.

2. Xie L, Zhou J, Zhang S, Chen Q, Lai R, Ding W, Song C, Meng X and $\mathrm{Wu}$ J: Integrating microRNA and mRNA expression profiles in response to radiation-induced injury in rat lung. Radiat Oncol 9: 111, 2014.

3. Rube CE, Uthe D, Schmid KW, Richter KD, Wessel J, Schuck A, Willich $\mathrm{N}$ and Rube C: Dose-dependent induction of transforming growth factor beta (TGF-beta) in the lung tissue of fibrosis-prone mice after thoracic irradiation. Int $\mathbf{J}$ Radiat Oncol Biol Phys 47: 1033-1042, 2000.

4. Arpin D, Perol D, Blay JY, Falchero L, Claude L, Vuillermoz-Blas S, Martel-Lafay I, Ginestet C, Alberti L, Nosov D, et al: Early variations of circulating interleukin- 6 and interleukin-10 levels during thoracic radiotherapy are predictive for radiation pneumonitis. J Clin Oncol 23: 8748-8756, 2005.

5. Chiang CS, Liu WC, Jung SM, Chen FH, Wu CR, McBride WH, Lee CC and Hong JH: Compartmental responses after thoracic irradiation of mice: Strain differences. Int J Radiat Oncol Biol Phys 62: 862-871, 2005.

6. Rübe CE, Rodemann HP and Rübe C: The relevance of cytokines in the radiation-induced lung reaction. Experimental basis and clinical significance. Strahlenther Onkol 180: 541-549, 2004 (In German).

7. Dhainaut JF, Charpentier J and Chiche JD: Transforming growth factor-beta: A mediator of cell regulation in acute respiratory distress syndrome. Crit Care Med 31 (Suppl 4): S258-S264, 2003.

8. Kim JY, Kim YS, Kim YK, Park HJ, Kim SJ, Kang JH, Wang YP, Jang HS, Lee SN and Yoon SC: The TGF- $\beta 1$ dynamics during radiation therapy and its correlation to symptomatic radiation pneumonitis in lung cancer patients. Radiat Oncol 4: 59, 2009.

9. Chung EJ, Hudak K, Horton JA, White A, Scroggins BT, Vaswani S and Citrin D: Transforming growth factor alpha is a critical mediator of radiation lung injury. Radiat Res 182: 350-362, 2014.

10. Németh K, Leelahavanichkul A, Yuen PS, Mayer B, Parmelee A, Doi K, Robey PG, Leelahavanichkul K, Koller BH, Brown JM, et al: Bone marrow stromal cells attenuate sepsis via prostaglandin E(2)-dependent reprogramming of host macrophages to increase their interleukin-10 production. Nat Med 15: 42-49, 2009.

11. Shi Y, Hu G, Su J, Li W, Chen Q, Shou P, Xu C, Chen X, Huang Y, Zhu Z, et al: Mesenchymal stem cells: A new strategy for immunosuppression and tissue repair. Cell Res 20: 510-518, 2010.

12. Wang H, Yang YF, Zhao L, Xiao FJ, Zhang QW, Wen ML, Wu CT, Peng RY and Wang LS: Hepatocyte growth factor gene-modified mesenchymal stem cells reduce radiation-induced lung injury. Hum Gene Ther 24: 343-353, 2013.

13. Asmussen S, Ito H, Traber DL, Lee JW, Cox RA, Hawkins HK, McAuley DF, McKenna DH, Traber LD, Zhuo H, et al: Human mesenchymal stem cells reduce the severity of acute lung injury in a sheep model of bacterial pneumonia. Thorax 69: 819-825, 2014.

14. Devaney J, Horie S, Masterson C, Elliman S, Barry F, O'Brien T, Curley GF, O'Toole D and Laffey JG: Human mesenchymal stromal cells decrease the severity of acute lung injury induced by $E$. coli in the rat. Thorax 70: 625-635, 2015 .

15. Rao $X$, Huang $X$, Zhou $Z$ and Lin $X$ : An improvement of the $2^{\wedge}$ (-delta delta CT) method for quantitative real-time polymerase chain reaction data analysis. Biostat Bioinforma Biomath 3: 71-85, 2013.

16. Saether EE, Chamberlain CS, Leiferman EM, Kondratko-Mittnacht JR, Li WJ, Brickson SL and Vanderby R: Enhanced medial collateral ligament healing using mesenchymal stem cells: Dosage effects on cellular response and cytokine profile. Stem Cell Rev 10: 86-96, 2014.

17. Yavagal DR, Lin B, Raval AP, Garza PS, Dong C, Zhao W, Rangel EB, McNiece I, Rundek T, Sacco RL, et al: Efficacy and dose-dependent safety of intra-arterial delivery of mesenchymal stem cells in a rodent stroke model. PLoS One 9: e93735, 2014.

18. Fukuda Y, Horie N, Satoh K, Yamaguchi S, Morofuji Y, Hiu T, Izumo T, Hayashi K, Nishida N and Nagata I: Intra-arterial transplantation of low-dose stem cells provides functional recovery without adverse effects after stroke. Cell Mol Neurobiol 35: 399-406, 2015. 
19. Kean TJ, Lin P, Caplan AI and Dennis JE: MSCs: Delivery routes and engraftment, cell-targeting strategies, and immune modulation. Stem Cells Int 2013: 732742, 2013

20. Li Z, Hu X, Mao J, Liu X, Zhang L, Liu J, Li D and Shan H: Optimization of mesenchymal stem cells (MSCs) delivery dose and route in mice with acute liver injury by bioluminescence imaging. Mol Imaging Biol 17: 185-194, 2015.

21. Song YS, Lee HJ, Doo SH, Lee SJ, Lim I, Chang KT and Kim SU: Mesenchymal stem cells overexpressing hepatocyte growth factor (HGF) inhibit collagen deposit and improve bladder function in rat model of bladder outlet obstruction. Cell Transplant 21: 1641-1650, 2012.

22. Zhang K, Yang S, Zhu Y, Mo A, Zhang D and Liu L: Protection against acute radiation-induced lung injury: A novel role for the anti-angiogenic agent Endostar. Mol Med Rep 6: 309-315, 2012.

23. Kim MD, Kim SS, Cha HY, Jang SH, Chang DY, Kim W, Suh-Kim H and Lee JH: Therapeutic effect of hepatocyte growth factor-secreting mesenchymal stem cells in a rat model of liver fibrosis. Exp Mol Med 46: e110, 2014.

24. Ortiz LA, Gambelli F, McBride C, Gaupp D, Baddoo M, Kaminski N and Phinney DG: Mesenchymal stem cell engraftment in lung is enhanced in response to bleomycin exposure and ameliorates its fibrotic effects. Proc Natl Acad Sci USA 100 8407-8411, 2003.

25. Ooi YY, Dheen ST and Tay SS: Paracrine effects of mesenchymal stem cells-conditioned medium on microglialcytokinesexpression and nitric oxide production. Neuroimmunomodulation 22 233-242, 2015.

26. Cho KS, Park MK, Kang SA, Park HY, Hong SL, Park HK, Yu HS and Roh HJ: Adipose-derived stem cells ameliorate allergic airway inflammation by inducing regulatory $\mathrm{T}$ cells in a mouse model of asthma. Mediators Inflamm 2014: 436476, 2014.
27. Curley GF, Hayes M, Ansari B, Shaw G, Ryan A, Barry F, O'Brien T, O'Toole D and Laffey JG: Mesenchymal stem cells enhance recovery and repair following ventilator-induced lung injury in the rat. Thorax 67: 496-501, 2012.

28. Zhao MM, Cui JZ, Cui Y, Li R, Tian YX, Song SX, Zhang J and Gao JL: Therapeutic effect of exogenous bone marrow derived mesenchymal stem cell transplantation on silicosis via paracrine mechanisms in rats. Mol Med Rep 8: 741-746, 2013.

29. Lee JW, Krasnodembskaya A, McKenna DH, Song Y, Abbott J and Matthay MA: Therapeutic effects of human mesenchymal stem cells in ex vivo human lungs injured with live bacteria. Am J Respir Crit Care Med 187: 751-760, 2013.

30. Kotton DN, Ma BY, Cardoso WV, Sanderson EA, Summer RS, WilliamsMCandFine A:Bonemarrow-derived cells asprogenitors of lung alveolar epithelium. Development 128: 5181-5188, 2001.

31. Prockop DJ: Marrow stromal cells as stem cells for nonhematopoietic tissues. Science 276: 71-74, 1997.

32. Ye J and Gimble JM: Regulation of stem cell differentiation in adipose tissue by chronic inflammation. Clin Exp Pharmacol Physiol 38: 872-878, 2011.

33. François S, Bensidhoum M, Mouiseddine M, Mazurier C, Allenet B, Semont A, Frick J, Saché A, Bouchet S, Thierry D, et al: Local irradiation not only induces homing of human mesenchymal stem cells at exposed sites but promotes their widespread engraftment to multiple organs: A study of their quantitative distribution after irradiation damage. Stem Cells 24: 1020-1029, 2006 